\title{
A case of idiopathic pulmonary fibrosis treated with clarithromycin
}

\author{
Masashi Ohe $^{1, *}$ and Satoshi Hashino ${ }^{2}$ \\ ${ }^{1}$ Department of General Medicine, Hokkaido Social Insurance Hospital, Sapporo, Japan \\ ${ }^{2}$ Hokkaido University, Health Care Center, Sapporo, Japan
}

\begin{abstract}
We report a case of idiopathic pulmonary fibrosis (IPF) treated successfully using clarithromycin (CAM). A 64-year-old male patient suffering from IPF which had been diagnosed at 59 years old, presented with slowly progressive dyspnea. Until this time, his condition had been almost stable. He was diagnosed with exacerbation of IPF, based on exacerbation of lung ausculation, O2 saturation by pulse oxymetry, Krebs von den Lungen 6 levels and chest computed tomography findings of reticular opacities. He was successfully treated using CAM in expectation of its anti-inflammatory effects. This case shows that treatment using CAM may be effective in some cases of IPF.
\end{abstract}

Key Words: Idiopathic pulmonary fibrosis, Clarithromycin

\section{Introduction}

Idiopathic pulmonary fibrosis (IPF) is progressive and fatal lung disease. Recent study suggested pirfenidone might be effective in slowing the decline of lung function on early-stage IPF persons (1). However, despite multiple recent clinical trials, no definite therapy other than lung transplantation is known to alter survival (2). Macrolides (Mac) have not only anti-bacterial activity but also immunomodulatory effects including anti-inflammatory effects. Successful Mac treatments of diffuse panbronchiolitis, cystic fibrosis, chronic obstructive pulmonary disease and bronchial asthma have been reported. Recently, a beneficial response to clarithromycin (CAM) in a patient with airway-centered interstitial fibrosis was reported (3). Here, we also report a case of IPF patient successfully treated using CAM.

\section{Case report}

A 64-year-old male patient suffering from IPF, visited our hospital with slowly progressive dyspnea over 2 months. He complained of neither cough nor sputum. At 59 years old, he had been diagnosed with IPF, based on laboratory findings, chest computed tomography (CT) findings and so on. Because $\mathrm{O}_{2}$ saturation by pulse oxymetry $\left(\mathrm{SpO}_{2}\right)$, laboratory findings such as C-reactive protein (CRP), lactate dehydrogenase (LDH), and Krebs von den Lungen (KL)-6 levels which are known to indicate the activity of interstitial pneumonia (4), and CT findings remained almost unchanged, he did not receive any treatment. Six months before this episode of dyspnea, $\mathrm{SpO}_{2}$ indicated $94 \%$ in room air at rest. CRP, $\mathrm{LDH}$, and KL-6 levels were $0.2 \mathrm{mg} / \mathrm{dL}$ (normal range $<0.3$ $\mathrm{mg} / \mathrm{dL}$ ), $217 \mathrm{U} / \mathrm{L}$ (normal range $<229 \mathrm{U} / \mathrm{L}$ ) and $965 \mathrm{U} / \mathrm{mL}$ (normal range $<500 \mathrm{U} / \mathrm{mL}$ ), respectively. CT revealed reticular opacities accompanied by honeycombing (Figure 1a). On this visit, exacerbated fine crackles were audible mainly in the right lower lung fields. $\mathrm{SpO}_{2}$ decreased to $92 \%$ in room air at rest. CRP, LDH, and KL-6 levels increased to $0.51 \mathrm{mg} / \mathrm{dL}, 236$ $\mathrm{U} / \mathrm{L}$ and $1,503 \mathrm{U} / \mathrm{mL}$, respectively. Chest $\mathrm{CT}$ and chest high resolution (HR) CT revealed exacerbated reticular opacities in the right middle and left lower lobes accompanied by honeycombing (Figures 1b, 2a). $\beta$-D-glucan levels were $3.0 \mathrm{pg} / \mathrm{mL}$ (normal range $<20.0 \mathrm{pg} / \mathrm{mL}$ ), and immunoglobulin $\mathrm{M}$ antibodies to cytomegalovirus and Mycoplasma pneumoniae were both negative. Finally, he was diagnosed with exacerbation of IPF, not pulmonary infections. After obtaining informed consent, he was treated with CAM (800 $\mathrm{mg} /$ day) in expectation of its anti-inflammatory effects, as an alternative to pirfenidone, which was too expensive for him. Six months after this treatment, his symptom improved considerably 


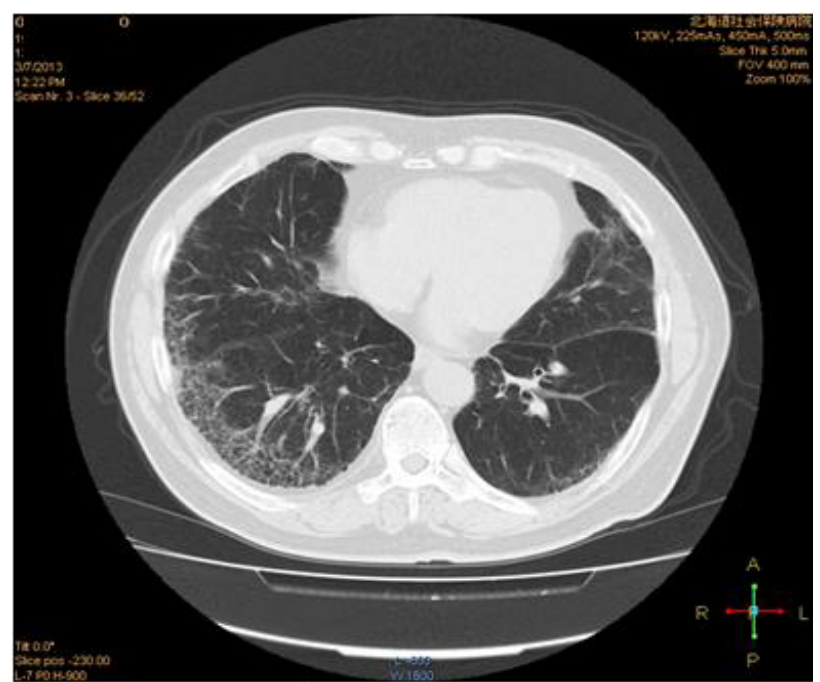

Fig. 1a. Chest computed tomography (CT) showed reticular opacities accompanied by honeycombing 6 months before this episode of dyspnea.

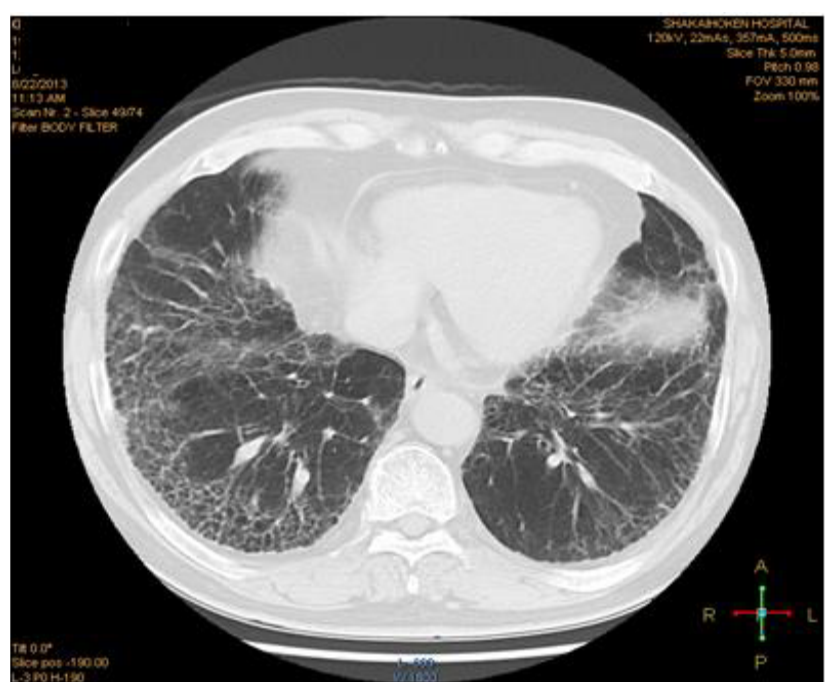

Fig. 1b. Chest CT showed exacerbated reticular opacities in the right middle and left lower lobes accompanied by honeycombing on this episode of dyspnea.

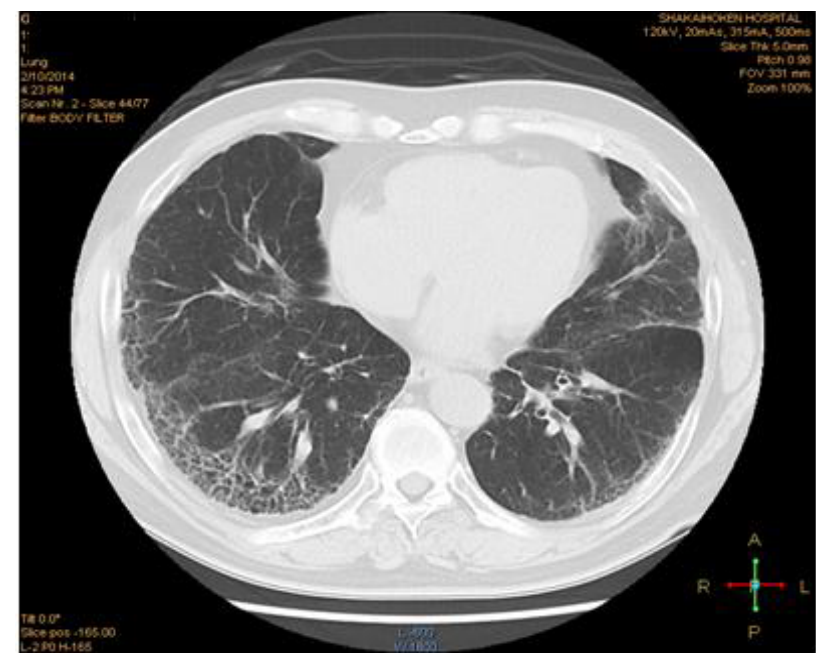

Fig. 1c. Chest CT showed considerably improved reticular opacities accompanied by honeycombing 6 months after clarithromycin (CAM) treatment.

with $\mathrm{SpO}_{2}$ in room air at rest, $\mathrm{CRP}, \mathrm{LDH}$ and $\mathrm{KL}$ 6 indicating $95 \%, 0.2 \mathrm{mg} / \mathrm{dL}, 213 \mathrm{U} / \mathrm{mL}$ and 986 $\mathrm{U} / \mathrm{mL}$, respectively. The considerable improvement of reticular opacities on chest CT and chest HRCT was recognized (Figure 1c, 2b). Long-term use of CAM caused slightly loose stool as a side effect.

\section{Discussion}

In the present case, the patient was diagnosed with exacerbation of IPF, not pulmonary infections. Regarding exacerbation of IPF, this episode of dyspnea was thought to be due to disease progression, not acute exacerbation, in consideration of slowly progressive dyspnea over 2 months and exacerbation of reticular opacities which were not superimposed on a background reticular or honeycomb pattern. The patient was successfully treated using CAM in expectation of its anti-inflammatory effects, not anti-bacterial activity.

Jouneau et al. (3) reported successful treatment of interstitial fibrosis with CAM (3). In the report, the patient was treated initially with prednisolone without any efficacy. During this treatment, the pulmonary function tests worsened. Therefore, CAM $(500 \mathrm{mg} /$ day for 1 month and then 250 $\mathrm{mg} /$ day) was added to prednisolone. As a result,

East J Med Volume:21, Number:1, January-March/2016 


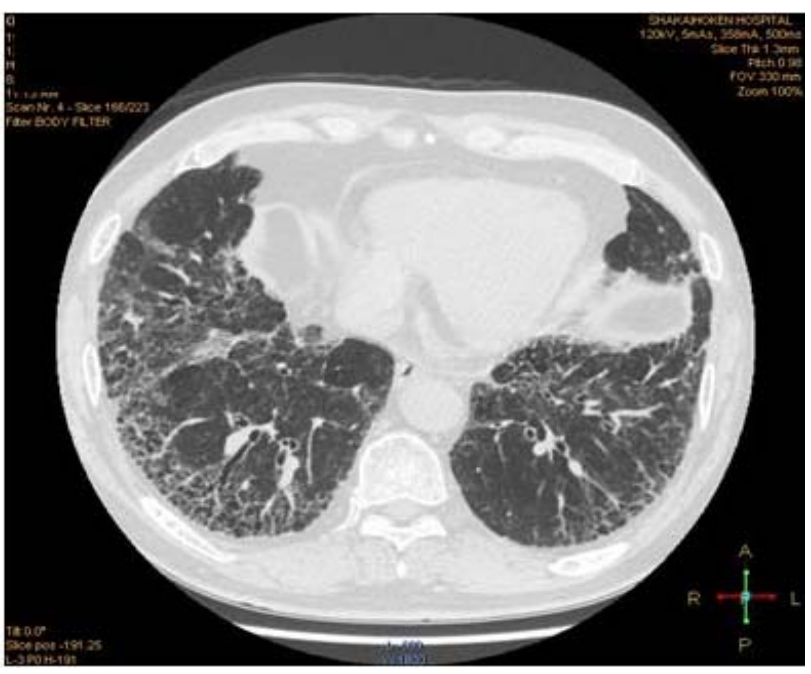

Fig. 2a. Chest high resolution (HR) CT showed exacerbated reticular opacities in the right middle and left lower lobes accompanied by honeycombing on this episode of dyspnea.

CAM stopped lung function decline with no change in chest CT. But, in our present case, reticular opacities on chest $\mathrm{CT}$ and chest HRCT improved considerably. The difference in improvement on CT findings between two cases might be related to prescribed CAM dosage.

Regarding immunomodulatory effects of Mac, Mac have an inhibitory effect on proinflammatory cytokines, such as tumor necrosis factor (TNF)alpha, interleukin (IL)-1 and nitric oxide. On the other hand, Mac increase the expression of IL-10, which protects host tissue during an infection by inhibiting the synthesis of interferon-gamma by both $\mathrm{T}$ cells and natural killer cells and the synthesis of nitric oxide by macrophages. Mac accelerate apoptosis of neutrophils and other leukocytes and the removal of cell debris by macrophages (5). Chen et al. (6) reported ameliorating effects of erythromycin on bleomycin-induced fibrosis through suppression of TNF-alpha and platelet derived growth factor as well as the inhibition on accumulation of inflammatory cells in the lung. Based on these facts, the effectiveness of CAM for IPF in the present case might be derived from abovementioned anti-inflammatory effects.

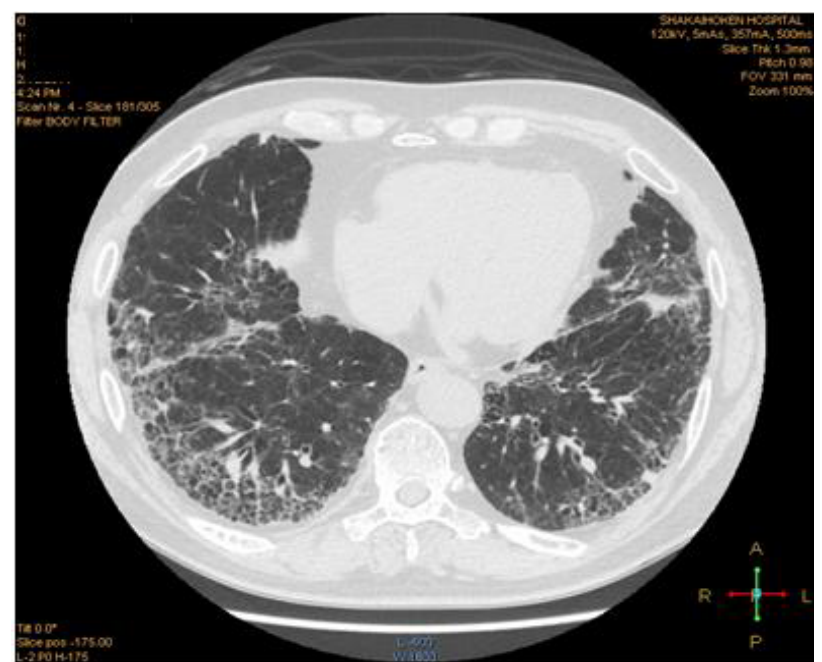

Fig. 2b. Chest HRCT showed considerably improved reticular opacities accompanied by honeycombing 6 months after CAM treatment.

Because only two cases including the present case have been reported, further research is necessary before CAM treatment can be adopted.

Conflict of interest: No potential conflict of interest relevant to this article is reported.

\section{References}

1. Taniguchi H, Ebina $M$, Kondoh $Y$, et al. Pirfenidone in idiopathic pulmonary fibrosis. Eur Respir 2010; 35: 821-829.

2. Kim YH and Kwon SS. Interstitial lung diseases: Respiratory reviews of 2013. Tuberc Respir Dis 2013; 75: 47-51.

3. Jouneau S, Kerjouan M, Caulet-Maugendre S, et al. Clarithromycin stops lung function decline in airway-centered interstitial fibrosis. Respiration 2013; 85: 156-159.

4. Kohno N. Serum marker KL-6/MUC1 for the diagnosis and management of interstitial pneumonitis. J Med Invest 1999; 46: 151-158.

5. Hügle T. Immunology of fibrotic lung disease: managing infections whilst preventing autoimmunity? J Inflam Res 2011; 4: 21-27.

6. Chen B, Jiang L, Zhao W, Yu R, Hou XM. Ameliorating effect of erythromycin on bleomycin-induced pulmonary fibrosis: role of alveolar macrophage activation and cytokine release. Respoil 1997; 2: 151-155. 